\title{
Turbulent flame propagation in corn dust clouds formed in confined and open spaces
}

\author{
Vijaya Kumar Cheeda ${ }^{1}$
}

Received: 4 March 2020 / Accepted: 8 July 2020 / Published online: 23 July 2020

(c) Springer Nature Switzerland AG 2020

\begin{abstract}
Flame propagation stages in a corn dust cloud in a large room size confinement investigated. The clouds ignited by a weak heat source. The mass of the dust identified to be influenced the flame acceleration. Dust in the clouds burnt rapidly through several fluid dynamic mechanisms. Four types of instabilities contributed to fast flame development. Landau-Darrieus and Kelvin-Helmholtz instabilities increased the flame speed in the initial stages of the fireball. In the intermediate and final stages, corrugated and the wrinkled flame structure originated through Rayleigh-Taylor instabilities. In addition to flow aspects and instabilities, the shock waves also enhanced the flame speed. The shock overpressure measured with a dynamic pressure sensor. These shock waves induced Richtmyer-Meshkov instability in the fireball and developed turbulent flames. Density gradients across the fireball (due to higher temperature gradients) caused perturbations. Further, this gradient increased by the shock waves. The crosswind speed through the confinement windows was slower, therefore it has not enhanced the flame speed. Corn dust dispersed quickly to a wider area by the shock waves, established large dust clouds, and lead to explosions. The maximum surface temperature of the fireball predicted as $1384 \mathrm{~K}$. The preheat zone around the fireball identified by an image processing tool. The turbulence parameters at the fireball obtained from qualitative and quantitative methods and analyzed. The clouds of a small quantity of corn dust lead to the formation of larger fireballs due to higher kinematic viscosity than the energy dissipation rate.
\end{abstract}

Keywords Dust cloud explosion · Hydrodynamic instability in dust cloud explosions · Turbulent flame · Fire accidents and Fire safety

\begin{tabular}{ll}
\multicolumn{2}{l}{$\begin{array}{l}\text { List of symbols } \\
\text { LDI (DLI) }\end{array}$} \\
$\begin{array}{ll}\text { Landau-Darrieus instability also referred to as } \\
\text { Darrieus-Landau instability }\end{array}$ \\
KHI & Kelvin-Helmholtz instability \\
RTI & Rayleigh-Taylor instability \\
RMI & Richtmyer-Meshkov instability \\
DAQ & Data acquisition system \\
FPS & Frames per second \\
$R_{e}$ & Reynolds number \\
$D_{a}$ & Damköhler number \\
$K_{a}$ & Karlovitz number \\
$p_{D}$ & Dynamic pressure \\
$\rho$ & Fluid density \\
$\mathrm{u}^{\prime}$ & Turbulent intensity, velocity component
\end{tabular}

$\begin{array}{ll}\mathrm{L}_{T} & \text { Integral turbulent length scale } \\ \delta_{L} & \text { Laminar flame thickness } \\ S_{L} & \text { Laminar flame speed } \\ \mathrm{MS} / \mathrm{s} & \text { Mega samples per second } \\ \mathrm{ms} & \text { Millisecond } \\ \mathrm{g} & \text { Gram } \\ \mu \mathrm{m} & \text { Micrometre }\end{array}$

\section{Introduction}

In the processing industries, dust cloud explosions are one of the classified accidents. Improper handling of flours like corn, wheat, sugar, etc caused major fire accidents in the

Vijaya Kumar Cheeda, vijaya_cheeda_chanti@yahoo.co.in | 'DDESC, Tirupati, Andhra Pradesh 517 101, India. 
past. Similarly, mishandling of industrial consumables such as powder of alumina, magnesium, etc has led to fire hazards. Solid particles of these substances form a dust cloud in the atmospheric air. Such dust clouds in certain conditions had lead to fire explosions. Such hazards have been resulting in more often since the consumption of these items is increasing with industrial demands. Therefore there is an urgent need in controlling this accident. So, to minimize such events, dust cloud formation has to be controlled. Consequently, more attention was drawn from a safety point of view especially in the rapid production, transportation, and storage of these combustible substances. In the past two centuries, many lives have been lost and had happened great economic loss during these explosions.

In the food processing industries, such incidents took place especially in wheat flour mills, malt factories, redchili grinding mills. Also, dust particles in coal mines and pyrotechnic industries could form ignitable clouds which have been causing fire accidents every year. Such incidents took place at the Imperial sugar refinery at Port Wentworth Georgia in 2008 [1] and Mont Blanc tunnel (1999) due to dispersion of sugar powder and flour of margarine respectively. Similarly, coal dust and methane gas explosion in Dhanbad Dhori colliery in India (1965) caused a death toll of 268 miners and injured many. For this reason, the fire raged several weeks in the mine tunnels. Likewise, another coal dust explosion in Chasnala colliery India (1972) has taken the lives of 372 miners. This event has also destroyed several houses in neighboring villages. An explosion of cornstarch at the Douglas Starch Works Plant, Cedar Rapids, IA (United States) [2] has led to a heavy loss of life.

A carton synthetic material spreading was found to be the cause for the blaze set at Baku metro station in Azerbaijan (1995). Likewise, a fire disaster at Washburn A Mill in Minneapolis (1878) was the reason for the loss of many lives. Perhaps, this was the first reported event regarding the dust explosion in the nineteenth century. When dispersed in air, these materials could easily explode under certain conditions $[3,4]$. The dust cloud can be easily ignited by weak ignition sources like a lit-up cigarette, matchstick, gas torch, or air heaters [5-8]. The concentration of the dust is another measurable factor that is vital in initiating the blaze. Apart from the above factors, pressure in the confinement, velocity of wind and environment temperature, etc can also play a significant role in igniting the dust charge. Therefore, finding the susceptibility of the explosion of these particles when interacted with a weak heat source is set to be the first objective of the present study. Therefore, an electric heating coil having less energy was chosen to lit-up the cloud. The second objective is that the minimum ignitable cloud particle concentration or dust sample quantity. Third, studying the effects of turbulence, shock wave, and instabilities on the development of fireballs containing low dust quantities. The test setup, experiment procedure, and image processing are explained in the methodology section. The results are comprehensively elaborated in the later section. Subsequently, conclusions are asserted. Thereafter, ongoing and future work is stated. At the end of the article, an "Appendix" provided to indicate the image processing results for the prediction of the preheat zone around the dust fireball.

\section{Methodology}

A large room of $1500 \mathrm{~m}^{3}$ volume with windows of $2.2 \mathrm{~m}^{2}$ area had chosen for conducting the proposed experiments. This test enclosure is having an inner ceiling height of $5.67 \mathrm{~m}$. The windows of this test space were arranged to allow the atmospheric wind flow and this wind velocity was approximately $1.67 \mathrm{~m} / \mathrm{s}$. The test space primarily consisted of dust injection and ignition systems, a data acquisition system, and a high-speed camera. The dust injection was done with compressed air through a cylindrical steel canister which has $50 \mathrm{~mm}$ diameter and $120 \mathrm{~mm}$ long. Air pressure in the canister had maintained as 4.0 bar (absolute) until complete injection achieved, and the injection pressure was controlled by a gate valve. Once the dust canister is emptied, the gate valve has been closed. The compressor has a maximum delivery pressure capacity of 15 bar and the reservoir can store $0.5 \mathrm{~m}^{3}$ of compressed air at the maximum pressure. The test samples were prepared with commercially produced corn flour. The samples were measured using an electronic weighing machine. Tests were conducted for dust cloud containing flour of $0.5,2,5,7,10$, and $15 \mathrm{~g}$. The particle size of the cornflour is between 2 and $17 \mu \mathrm{m}$. The canister outlet was positioned vertically towards a heating coil as shown in Fig. 1, thus the jet of dust could pass through the heated coil.

An electric flat spiral coil made of nichrome alloy was employed as an igniter. Outer and inner diameters of the coil are $150 \mathrm{~mm}$ and $30 \mathrm{~mm}$ respectively and its total heat power is $500 \mathrm{~W}$. It becomes heated with an AC supply of $230 \mathrm{~V}$ and $2.18 \mathrm{~A}$. The coil steady-state temperature reached in five minutes. After reaching the steady-state condition, the ignition energy that liberated from the hot coil in $10 \mathrm{~ms}$ is $5 \mathrm{~J}$. This energy is higher than the cornflour minimum ignition energy $(20 \mathrm{~mJ})$. It can be observed in the results that the ignition is taking place in the first 10 $\mathrm{ms}$. At the center of the heater coil surface temperature had been measured (with a C-type thermocouple) as 483 ${ }^{\circ} \mathrm{C}(756.16 \mathrm{~K})$ and it is $350{ }^{\circ} \mathrm{C}(623.16 \mathrm{~K})$ at the edge when reached to steady-state. When the cloud contains corn dust more than $1 \mathrm{~g}$, then its ignition temperature is about 
$420{ }^{\circ} \mathrm{C}(693.16 \mathrm{~K})$ [9]. The temperature of the coil is well above the self-ignition temperature of corn dust.

The flame evolution and transition to different stages were recorded using a high-speed camera made by CASIO and the camera model is Exilim EX-FH20. The camera resolution is 9.1 megapixels and can operate at an exposure time of $25 \mu \mathrm{s}$ for each frame. The camera has a maximum frame rate capacity of 1000 FPS in video mode. In the present investigation, the frame rate is fixed at 1000 FPS. The camera has an Exilim lens and its focal length can be changed from 4.6 to $92 \mathrm{~mm}$. An image was processed in Matlab for finding the preheat zone and the result is given in the "Appendix". The dynamic pressure of the explosions in the test space was measured with the help of a piezoelectric transducer and two-channel data acquisition system (DAQ). The dynamic pressure sensor is mounted on a column at a distance of $1 \mathrm{~m}$ from the center of the coil. It can pick-up any disturbance caused in the ambient medium around the test set-up. The noise traveling through the ground and room structure was isolated with an elastic damper placed between the sensor and its mounting. The DAQ has $48 \mathrm{MHz}$ bandwidth and its maximum real-time sampling rate is $48 \mathrm{MS} / \mathrm{s}$. Signal time step is set as 1-ms for pressure measurements in this study.

The dynamic pressure data used to determine the fluctuating turbulent velocity component $\mathrm{u}^{\prime}$ and quantify turbulent combustion regimes via Reynolds number (Re). The turbulence factors such as Damköhler number (Da), Karlovitz number $(\mathrm{Ka})$ are calculated using the image data. The pressure wave traveling from the fireball is considered to be traveling in the incompressible fluid around the fireball, since the air velocity around the fireball is very low (flow Mach number is much less than 0.3). Therefore the variation of $\mathrm{u}^{\prime}$ on the flame front and dynamic pressure are proportionate to each other. They can be related as $p_{D}=\frac{\rho\left(u^{\prime}\right)^{2}}{2}$. Dynamic pressure data was also used to find the arrival of pressure waves upon the explosion of the cloud. The flame properties such as speed, thickness are obtained from the high-speed images. In addition to the flow-flame interaction, dynamics in various regimes (laminar and turbulent) via Landau-Darrieus instability (LDI), Kelvin-Helmholtz instability (KHI), Rayleigh-Taylor instability (RTI), and Richtmyer-Meshkov instability (RMI), the influence of pressure waves on the flame development is also studied.

Measuring the temperature of dust fireball (it is expanding axially and radially and while expanding, its space coordinates are random) in conventional quantitative methods is difficult. The thermocouple can not be used to measure fireball temperature. Since the available thermocouple dynamic response time is $0.75 \mathrm{~s}$. The thermocouple response time should be well below $10 \mathrm{~ms}$ to measure the instantaneous temperature in the fireball. Besides, holding these instruments in the fireball would also be unfeasible due to continuously accelerating turbulent flame. Moreover, the error induced in the measurements would also be higher. Because of these limitations, surface temperature of the fireball is measured in a qualitative method. The image pixel intensity values obtained (by image analysis with Matlab software) are used to calculate the surface temperature on the vertical axis of the fireball. For this, a pixel intensity should be known where surface temperature is also known. For this, an image was recorded in the location where the surface temperature of the hot coil was measured using the thermocouple (which is 756.16 $\mathrm{K})$. From this image, reference pixel intensity had been calculated and it is 130 . Thereafter, a ratio of pixel intensities between the location on the central axis of the fireball and the known temperature location on the hot coil was calculated. The pixel intensity ratio was multiplied with the hot coil surface temperature to calculate the surface temperature of the fireball at that specific location. For these predictions, the hot coil image and fireball images were converted into grayscale so that the pixel values would be consistent. Test results are arranged in order of minimum to maximum dust samples and observations are discussed categorically.

\section{Results and discussion}

The injected dust upon opening the gate valve had been formed as a cloud in a shorter time than the reactions would begin. The cloud volume is not going to be constant for each sample. The high momentum of dust makes the dust particles spread in all directions. The injection pressure and mass of dust in the canister decide the final cloud volume. The dust cloud volume had been calculated (from the image pixel coordinates) after $10 \mathrm{~ms}$ of injection beginning. This volume is assumed as a truncated cone. Calculated cloud volume and concentration data for each sample are given in Table 1. It can be noticed that the higher mass of dust dispersed to high volume and thus its concentration is moderate. This contributes to the dust particles interacting with a large amount of the surrounding air molecules. Here, a low mass sample and high

Table 1 Volumetric properties of corn dust cloud samples

\begin{tabular}{lll}
\hline Dust sample $(\mathrm{g})$ & $\begin{array}{l}\text { Dust cloud volume } \times 10^{-3} \\
\left(\mathrm{~m}^{3}\right)\end{array}$ & $\begin{array}{l}\text { Dust concentra- } \\
\text { tion } \times 10^{-3}\left(\frac{\mathrm{kg}}{\mathrm{m}^{3}}\right)\end{array}$ \\
\hline 2 & 27.4 & 73 \\
5 & 10.3 & 485 \\
7 & 23.2 & 301 \\
10 & 70 & 143 \\
15 & 271 & 55 \\
\hline
\end{tabular}


mass sample dust concentration values are comparable. Therefore, It creates confusion in interpreting the flame and flow characteristics in terms of concentration, hence the mass of the dust sample is preferred to compare the results of test cases. The heater coil has not ignited the $0.5 \mathrm{~g}$ of the dust cloud even after several trials. In $2 \mathrm{~g}$ test case, a flame kernel formed near the hot surface of the coil, after $10 \mathrm{~ms}$ of injection. This kernel can be seen at the time step of $20 \mathrm{~ms}$ in Fig. 2. Thereafter, it becomes a laminar flame at $30 \mathrm{~ms}$. In the preliminary stage of flame development, wrinkles had formed on the flame surface by diffusion of surrounding air into the fireball and also due to the Landau-Darrieus instability (LDI). The wrinkles at the interface between the flame and surrounding air can be seen in the frames from 60 to $100 \mathrm{~ms}$ of Fig. 2. The flame spreads upward faster since buoyant hot gases also moving vertically at the same speed. It was found [10] that the buoyant premixed reactant gases in clouds favor explosions in confined and semiconfined spaces. A similar trend can be seen in the present study with corn dust clouds. This spreading flame becomes more turbulent due to continual interactions of flame and surrounding airflow at their interface.

In the dust mass of $5 \mathrm{~g}$, the laminar flame developed rapidly compared to the case of $2 \mathrm{~g}$. In this instance, the flame travels faster since the dust particles are higher. LDI at the interface can be observed in the frames between 60 and $130 \mathrm{~ms}$ in Fig. 3. In addition to LDI, jet expansion has created turbulence in the flow and it led to the formation of cellular structured flame on the outer surface of the fireball at $140 \mathrm{~ms}$ time frame. Later the entraining of surrounding air into jet core has caused non-steady vortex rings in the time steps between 150 and 210 ms. Further, this flame had spread outward until the dust in the cloud is consumed in the intervals from 150 to $230 \mathrm{~ms}$. The initial jet can also be seen expanding radially outwards.

The oscillations in the pressure signal (in $2 \mathrm{~g}$ sample test) are signifying the rapidly expanding combustion product gases (volumetric expansion of hot gases) in the fireball which are shown in Fig. 4a. The rapid fluctuations in the fireball began at $100 \mathrm{~ms}$ after the injection of dust in $5 \mathrm{~g}$ sample case. The shock waves are produced by the expanding fireball after $90 \mathrm{~ms}$ of the ignition of the dust in the cloud. The pressure data indicates that the quantity of dust in the cloud, as well as the rate of expansion of fireball (due to rapid combustion of particles), are responsible for the generation of the shock waves. The dynamic pressure in the test case of $5 \mathrm{~g}$ for a time span of $400 \mathrm{~ms}$ is shown in Fig. 4b.

The rate of pressure rise in the test case of $2 \mathrm{~g}$ is about $80 \mathrm{bar} / \mathrm{ms}$ and it is shown in Fig. $5 \mathrm{a}$, whereas it is about $1400 \mathrm{bar} / \mathrm{ms}$ at $200 \mathrm{~ms}$ (shown in Fig. 5b) with $5 \mathrm{~g}$ sample. The pressure oscillations with $5 \mathrm{~g}$, indicates multiple shock wave starting from 100 ms onwards. These shock waves are due to the rapid burning of dust pockets in the fireball in a time span of about $2 \mathrm{~ms}$. Sample calculations for the rate of pressure rise are given in Table 2 . In the literature [11], the maximum pressure rise is specified as 12 bar/s for pulverized corn cob dust concentration of 600 $\frac{\mathrm{g}}{\mathrm{m}^{3}}$. In the present work, in the confined regions, strong shock waves have been formed since the cloud contains more dust which increased the rate of reaction. Thus, wave strength also increased compared to the $2 \mathrm{~g}$ test. The local explosion overpressure reached to 3.4 bar (absolute) for 10 g sample (Fig. 4c).

In the unconfined region, high energy accumulation is required to generate strong shock waves. Maximum shock overpressure reached for coal dust explosion recorded as 3 to 6.5 bars [12]. It is about 6.78 bar in sugar dust explosion [13] with concentration of $1000 \frac{\mathrm{g}}{\mathrm{m}^{3}}$. In a $0.005 \mathrm{~m}^{3}$ chamber, maximum explosion pressure for corn dust is 7.9 bar [14] for concentration of $1000 \frac{\mathrm{g}}{\mathrm{m}^{3}}$. This pressure history elucidates that the shock wave pressure depends on the amount of dust in the cloud as well as the chamber volume. Small constant volume chambers would create high pressure. In the current investigation, unburnt dust in the fireball has dispersed in the vertical direction by the buoyant hot gases. The spherical shock waves (with an overpressure of $3.4 \mathrm{bar}$ ) around the fireball spreading the dust in three directions and also causing R-M instabilities at the interface. Therefore, the fireball is expanding in three dimensions and this phenomenon is observed in the images of $10 \mathrm{~g}$ sample case. In addition to the shock waves [15-17], the rarefaction waves can also disperse dust particles to wide-area.

When the cloud concentration is increased to $7 \mathrm{~g}$, the flame features have become distinct. The spirals of the heating coil act as an obstacle to the jet flow and this obstacle causes Kármáán vortex street. The Kármán vortex street causing vortex shedding which mixes the reactants

Table 2 Pressure and maximum rate of pressure rise, given as an example

\begin{tabular}{lll}
\hline Pressure (bar) & Time step dt (ms) & $\begin{array}{l}\text { Maximum rate of } \\
\text { pressure rise, } \frac{d p}{d t}(\mathrm{bar} / \\
\mathrm{ms})\end{array}$ \\
\hline-0.4706 & 0.001 & -1317.7 \\
0.8471 & 0.001 & 815.7 \\
0.0314 & 0.001 & 753 \\
-0.7216 & 0.001 & -753 \\
0.0314 & 0.001 & 0 \\
0.0314 & 0.001 & 219.6 \\
-0.1882 & 0.001 & -94.1 \\
-0.0941 & 0.001 & -94.1 \\
- & - & - \\
\hline
\end{tabular}


from all sides and leading to fast-flame between 80 and $240 \mathrm{~ms}$. In the later stages, the products and unburnt charge in the cloud core spread radially as well as in the jet direction. This causes the corrugated flame through the Rayleigh-Taylor instability (RTI). RTI caused due to the rapid expansion of low-density product gas against the high density surrounding air. The interplay between these gases led to the formation of vortices from 250 to $450 \mathrm{~ms}$. The cloud formation, kernel growth, laminar and initial stages of turbulent flame can be seen in Fig. 6 .

In $10 \mathrm{~g}$ concentration, the laminar flame became turbulent within $50 \mathrm{~ms}$ time from the ignition of the fireball. The flame front starts wrinkling and transits into turbulent flamelets. The flamelets all around the core spread radially from the core. The RTI accelerates the flamelets. In the second stage, rapid heat release has led to the formation of the shock wave, thus causing RM instability at the outer surface of the fireball between 120 and 360 ms. Further, in the last stages fireball break up into fragments after the consumption of the dust at $440 \mathrm{~ms}$. A large preheating zone above the flamelets can be seen at $220 \mathrm{~ms}$ in Fig. 7 and also outer core rotation between 240 and 360 $\mathrm{ms}$. Here, the maximum pressure behind the shock wave is 3.4 bar (Fig. 4c). The shock wave formed at the fireball augments the mixing of reactants by high-intensity turbulence through the slipstream. Across this slipstream velocity gradients produce turbulence. In the last case of $15 \mathrm{~g}$ of dust, a large fireball has formed. During the intermediate and final stages, longer turbulent scales are produced which can be observed in Fig. 8 between time intervals of 330 and $460 \mathrm{~ms}$. They are formed due to the fluctuations caused by the entrainment of hot gases from the jet. These turbulent scales accelerate the flame further and swirl up to the confinement ceiling of $5 \mathrm{~m}$ in height. The fluctuating velocity components cause a disturbance in the fireball. The velocity components are as high as $50 \mathrm{~m} / \mathrm{s}$. Higher oscillations in the flow distorted the flame surface which fragmented the flame surface into laminar flamelets.

The laminar flamelet speed $\left(S_{L}\right)$ is $0.5 \mathrm{~m} / \mathrm{s}$ for lower concentrations and $195.3 \mathrm{~m} / \mathrm{s}$ for $15 \mathrm{~g}$ of dust. The lower limit of laminar flamelet speed recorded in this work is in coincident to that quoted in the paper [18] of corn dust and fuel gas mixture explosions conducted in $1 \mathrm{~m}^{3}$ closed chamber, however, the upper limit is many times higher. Across the fireball maximum and minimum temperature, profiles are plotted with dust samples. A maximum temperature of $1384 \mathrm{~K}$ was reached with the dust sample of $7 \mathrm{~g}$ but this profile is almost constant for all the samples. Whereas the minimum temperature profile was varying between 721 and $1000 \mathrm{~K}$. The temperature profile is shown in Fig. 9.

The wrinkled and cellular flame development is observed as a common phenomenon in the dust clouds so far. The diffusion phenomena contributing to the fast flamelets in the second (or intermediate) stages. L-D instability is responsible for the development of wrinkled flames in the zone close to the laminar burning zone (Fig. 10) [19]. In large rooms upon ignition of a few grams of dust formed large fireballs. Ignition and laminar flame front development are mainly influenced by $\mathrm{K}-\mathrm{H}$ instability therefore wrinkles formed on the edges of the flame surface in the initial stages irrespective of the amount of the dust contained in the clouds. In the third stage, RTI and RMI are more influential in obtaining fast flames especially in the higher concentrations of the dust in the clouds (Fig. 11) and in these cases, large turbulent scales break into small scales which increases the flamelet speed. The turbulent scales formed is of size ranging from 0.63 to 1.39 $\mathrm{m}$.

Strong shock wave induces large density gradients and induces RM instability in the flow [20-24] in the premixed gases. Such waves are also seen to grow RM instabilities in the dust clouds (non-premixed reactants). An impulsive acceleration of flame has achieved by these growing perturbations and eventually created vortices at the fireball. This has led to an increased rate of reaction and further produced multiple shock waves. The RMI causes vorticity which has developed corrugations in the flame surface (Fig. 12). In turn, the vorticity production depends on gradients of density [25] in addition to pressure and temperature gradients across the jet field and the atmospheric air conditions. The higher pressure and density gradients in the flow field have induced large scale turbulent eddies. The orientation of pressure and density gradients is shown in Fig. 12. The growth of this turbulence depends on the energy liberated at the time of initial perturbations. Flame acceleration in the laminar regime predominantly depends on the reaction rate at that instance. When the reaction rate increased at a particular pocket of dust would produce perturbations. Since the increased reactions would also cause flow field gradients like density, pressure, and temperature across the particular local dust pockets and its neighboring regions. This initiates the turbulence which continues to increase its intensity until it self-sustains. In addition to the shock waves, the crosswind velocity also enhances the mixing of gases and dust. Here, the effect of crosswind is less influential since the atmospheric wind velocity is only $1.67 \mathrm{~m} / \mathrm{s}$. In the confined space, vorticity in the reactive dust flow with large concentrations of dust may also lead to detonation[26, 27].

The vortex stretching by RMI leads to the rapid development of the fireball in the open space (Fig. 13). This leads to faster acceleration[27] of the flame. In the open space, the fireball has grown to a height of $7.5 \mathrm{~m}$ with $15 \mathrm{~g}$ of corn dust. This large fireball can be seen in the time frame of $350 \mathrm{~ms}$ in Fig. 13. Therefore, the vortex stretching accelerates the flame in addition to instabilities. Along with the 
Table 3 Flame characteristics in the fireball in confinement

\begin{tabular}{|c|c|c|c|c|c|c|c|}
\hline $\begin{array}{l}\text { Sample } \\
\text { size (g) }\end{array}$ & $\begin{array}{l}\text { Turbulent } \\
\text { intensity } u^{\prime} \\
(\mathrm{m} / \mathrm{s})\end{array}$ & $\begin{array}{l}\text { Laminar flame } \\
\text { (flamelet) speed } S_{L} \\
(\mathrm{~m} / \mathrm{s})\end{array}$ & $\begin{array}{l}\text { Integral } \\
\text { length scale } \\
L_{T}(\mathrm{~m})\end{array}$ & $\begin{array}{l}\text { Laminar flame } \\
\text { thickness } \delta_{T} \times 10^{-6} \\
\text { (m) }\end{array}$ & $\begin{array}{l}\text { Turbulent Reynolds } \\
\text { number } R e_{T} \times 10^{5}\end{array}$ & $\begin{array}{l}\text { Damkohler } \\
\text { number } \\
D_{a} \times 10^{5}\end{array}$ & $\begin{array}{l}\text { Karlovitz } \\
\text { number } \\
K_{a} \times 10^{5}\end{array}$ \\
\hline 2 & 14.8 & 7.5 & 0.04 & 56.9 & 0.015 & 0.004 & 0.021 \\
\hline 5 & 0.37 & 0.34 & 1.39 & 6.18 & 2.45 & 2.0 & 2.5 \\
\hline 7 & 37.1 & 7 & 0.5 & 0.61 & 0.4 & 0.015 & 1.0 \\
\hline 10 & 13.57 & 9.3 & 0.52 & 0.45 & 0.1 & 7.86 & 0.2 \\
\hline 15 & 47 & 195.3 & 0.58 & 2.18 & 0.64 & 11.2 & 0.3 \\
\hline
\end{tabular}

Table 4 Temperature profile in axial direction of the fireball

\begin{tabular}{lcllll}
\hline Point & $\begin{array}{l}\text { Pixel intensity } \\
(\mathrm{x})\end{array}$ & $\begin{array}{l}\text { Reference pixel } \\
\text { intensity }(\mathrm{y})\end{array}$ & $\frac{x}{y}$ & $\begin{array}{l}\text { Reference tempera- } \\
\text { ture } R_{T}(\mathrm{~K})\end{array}$ & $\begin{array}{l}\text { Temperature at } \\
\text { point }{ }^{\prime} \mathrm{x}_{T} \times \frac{x}{y}(\mathrm{~K})\end{array}$ \\
\hline 1. & 7 & - & - & - & 310 \\
2. & 57 & 130 & 0.438 & 756 & 331 \\
3. & 67 & 130 & 0.515 & 756 & 389 \\
4. & 146 & 130 & 1.123 & 756 & 849 \\
5. & 117 & 130 & 0.9 & 756 & 680 \\
6. & 210 & 130 & 1.615 & 756 & 1221 \\
\hline
\end{tabular}

Fig. 1 Test setup showing dust injection and ignition systems, location of high speed camera and pressure transducer

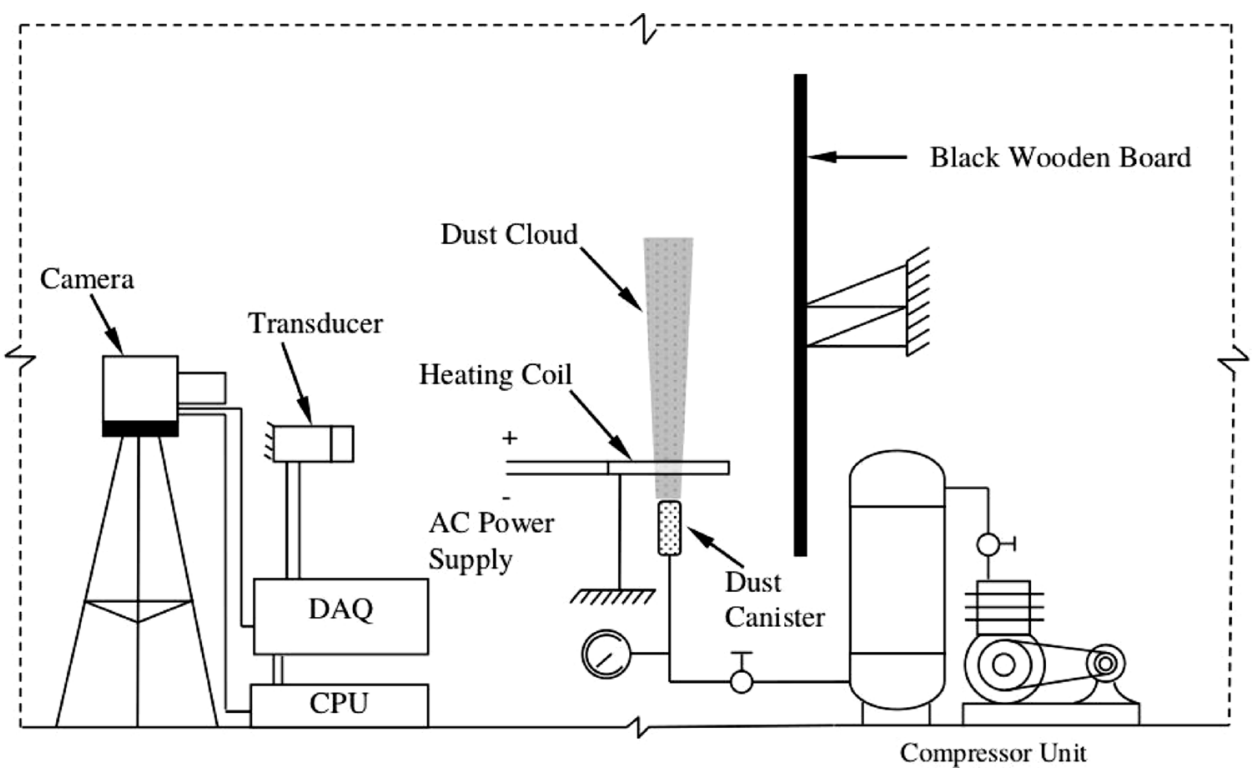

shock waves, the atmospheric wind also scatters the dust to a wider area.

R-T instabilities caused corrugated flame surface on the fireball as seen in Fig. 14 images 4 and 5 which results in rapid heat release. Thus the fireball expands faster over a wider area. Flame front rolled up on the vortex ring observed in image 3 of Fig. 14. The jet of compressed air and dust causes this vortex ring due to induced centripetal velocity on the entraining air towards the center of the jet. This vortex ring is seen much below the turbulent main flame. Thus the preheating is done and the reactions are initiated in the vortex ring itself. The expansion of product gas causes high-speed flow and this flow can form strong shock waves. Such strong shock waves can generate a vortex of about one-fourth of the confinement size[28, 29] due to RTI and RMI[30]. Therefore the flame spins across the duct until the fuel is consumed in the enclosure. The entraining air and dust mixture are reignited by the main combustion charge in the last stages. A non-uniform density gradients[31] in the dust clouds cause vortices similar to that of premixed gaseous flows. The jet of dust in the presence of shock waves (when these waves interact with 


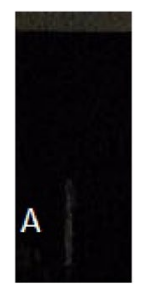

10

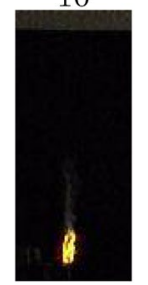

60

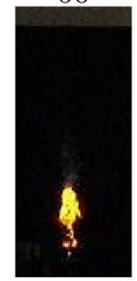

110

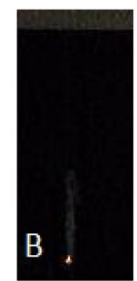

20

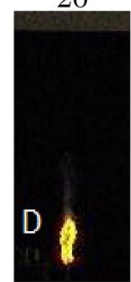

70

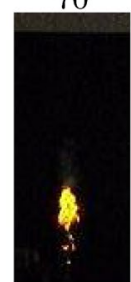

120

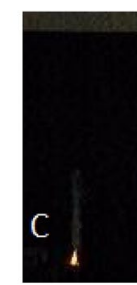

30

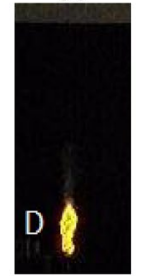

80

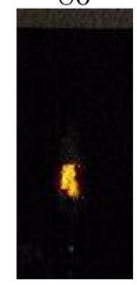

130

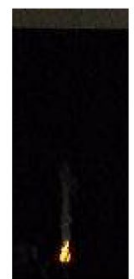

40

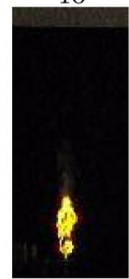

90

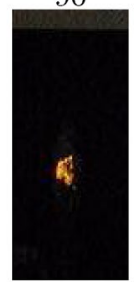

140

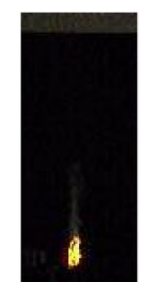

50

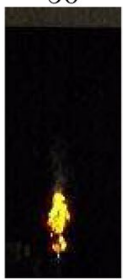

100

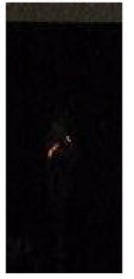

150
Fig. 2 Flame propagation in fireball containing $2 \mathrm{~g}$ of corn dust; $\mathbf{A}$ Dust cloud, B Flame kernal, C Laminar flame, D Wrinkled flame by L-D instability

dust, surrounding air, and flame surface) led to fast flames. L-D instability also causes acoustic waves[32] and this intensifies acceleration of the flame in the preliminary stages and leads to fast flame[33, 34]. The turbulent flame regimes are determined through flame and flow characteristics such as turbulent intensity, laminar flame (flamelet) speed, laminar flame thickness, integral length scale. The Reynolds number depicts that the flow in the fireball at the interface is turbulent. These values are given in Table 3. The different types of flame structures characterized are shown in the Borghi-Peters diagram in Fig. 15. Two non-dimensional ratios (one is integral turbulent length to laminar flame thickness $\left(\frac{L_{T}}{\delta_{L}}\right)$ and the second one is the turbulent intensity to laminar speed $\left.\left(\frac{u^{\prime}}{S_{L}}\right)\right)$ has classified the turbulent flame into four regimes. The turbulent parameters (maximum values in each case) indicate that turbulent intensity and laminar flame speed ratio is between 1 and 10 for all dust concentrations. Whereas the ratio of integral turbulent length to laminar flame thickness spreads for wider zones with values ranging from $10^{2}$ to $10^{5}$. These ratios show that the fireball falls into two flame regimes one is corrugated flamelets and the second one is wrinkled flamelets. The dust concentrations between 2 and $10 \mathrm{~g}$ yield corrugated flamelets in the fireball and a higher concentration of $15 \mathrm{~g}$ has wrinkled flamelets. Karlovitz number (Ka) and Damkohler numbers (Da) signify large kinematic viscosity and turbulence kinetic energy dissipation rate in the preheat zone and in the fireball. It shows that the Kolmogorov scale is smaller than the laminar flame thickness. The Kolmogorov scale is influenced by the large turbulence kinetic energy dissipation rate per unit mass, than the (local) kinematic viscosity of the gas in small dust samples. For large dust samples, the Ka is small, which means that the Kolmogorov scale is larges than in the other test cases. It tells that the turbulence kinetic energy dissipation is lower than the kinematic viscosity of the medium. Thus the turbulence is selfsustained for longer periods and hence the fireball spreads to the wide area.

\section{Conclusions}

Corn dust of small quantities in the cloud can form large fireballs. Dust of $5 \mathrm{~g}$ has formed fireball equivalent to the size of the confinement $(5 \mathrm{~m})$. L-D and $\mathrm{K}-\mathrm{H}$ instabilities contribute to increasing the velocity of flame in the fireball in the initial stage however the flame acceleration is slower. In the subsequent stages, the explosion of these dust clouds shows that high-pressure shock waves have been generated. These shocks were responsible for R-M instabilities through the density and pressure gradients. These instabilities intensify flame speed and break up the flame into flamelets. RTI and RMI result in vorticity at the interface of the fireball and the surrounding air. The vortex flow at the interface caused the curl in the flame front which increases the flame speed through the mixing of reactants. This phenomenon has been observed in all directions. Simultaneously, the entrainment of the hot gases into the cloud has contributed to the generation of large turbulent scales.

The instabilities accelerate the diffusion flame in the dust cloud similar to the diffusion combustion of liquid fuel droplets. Intense turbulence has achieved in large dust clouds through the shock waves in large rooms as well as in the open spaces. The maximum and minimum surface temperatures of the fireball are $1384 \mathrm{~K}$ and $721 \mathrm{~K}$ respectively. Since the confinement is larger than the dust cloud, high pressure would not be build up like in a constant volume chamber. However, the overpressure caused by the shock waves would increase the fireball size. Large fireballs in confinements are due to the self-sustained turbulence because of higher values of kinematic viscosity than kinetic energy dissipation rate.

A small quantity of ignitable dust can easily be dispersed by the circulating atmospheric air around the stockpiles of cornflour and also while transporting it. This scattered dust can be ignited by weak ignition sources and 
Fig. 3 Stages of flame propagation and its extinction in the fireball containing dust of $5 \mathrm{~g}$; A Radially expanding fireball, $\mathbf{B}$ Vortex rings
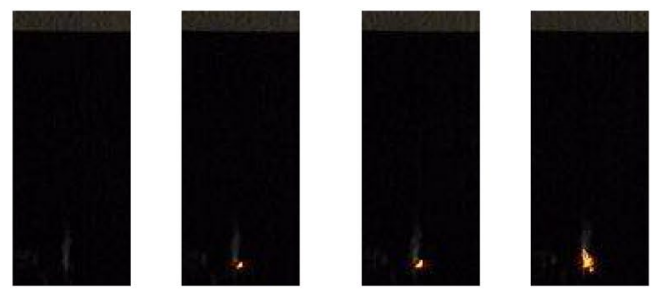

30

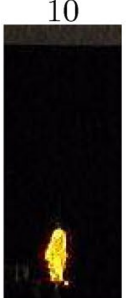

20

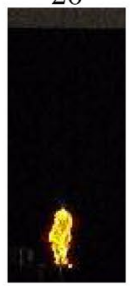

80

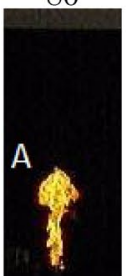

90

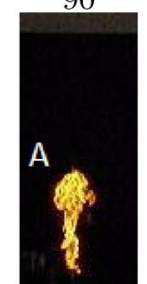

150
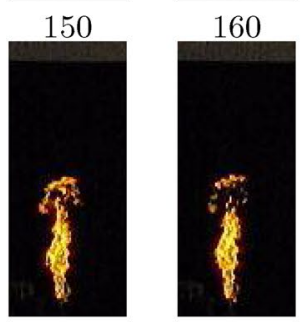

220

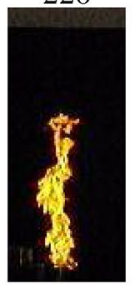

290
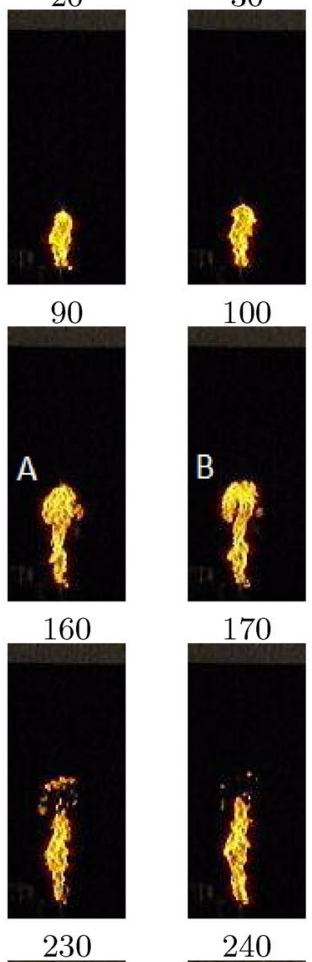

40

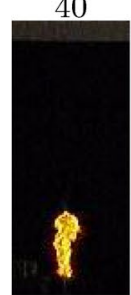

100

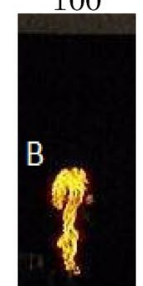

110

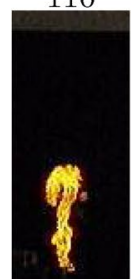

170

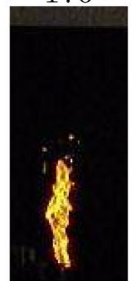

180

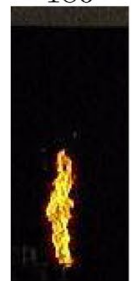

240

250

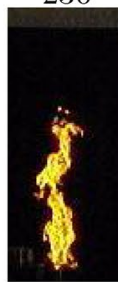

300

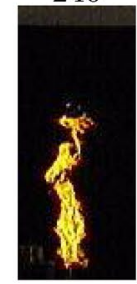

310

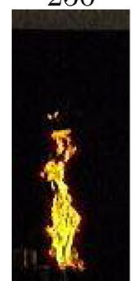

320

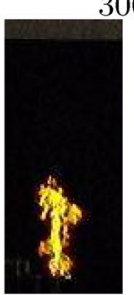

360
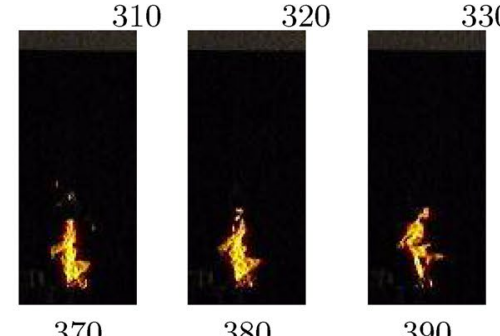

330
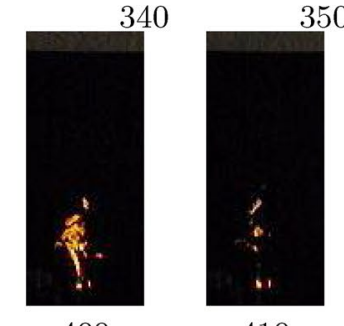

400

410

340

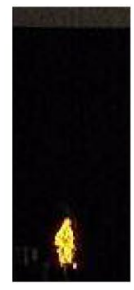

70

Cellular

flame

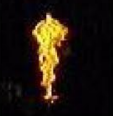

140

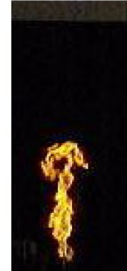

210
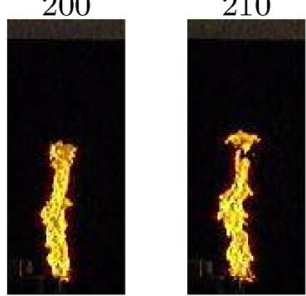

280

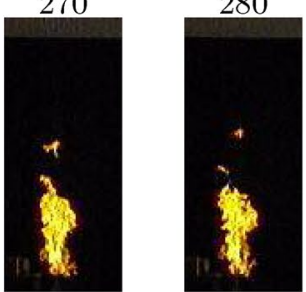

350 


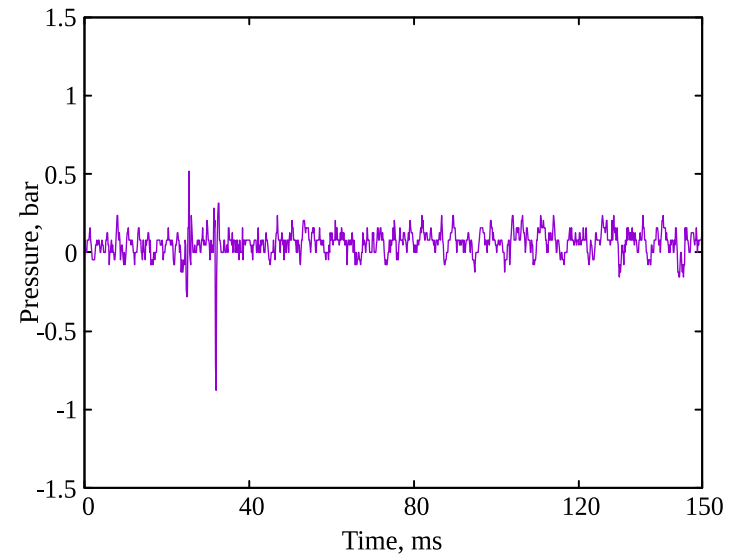

a 2 grams

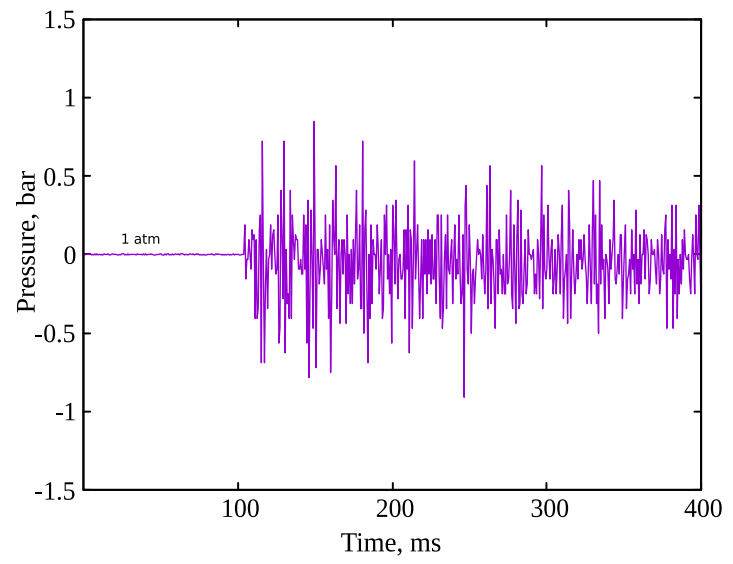

b 5 grams

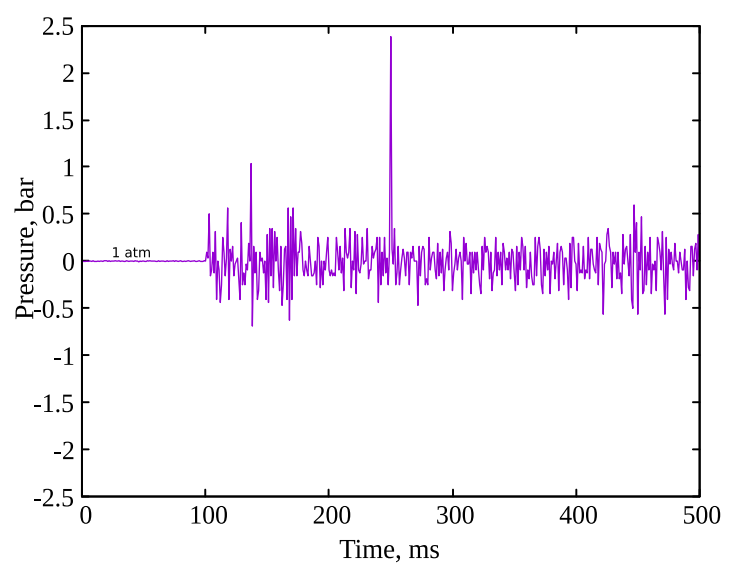

c 10 grams

Fig. 4 Dynamic pressure profile showing shock waves (compression waves) generation and their oscillations in the fireball formed in dust clouds of $\mathbf{a} 2 \mathrm{~g}, \mathbf{b} 5 \mathrm{~g}$ and $\mathbf{c} 10 \mathrm{~g}$

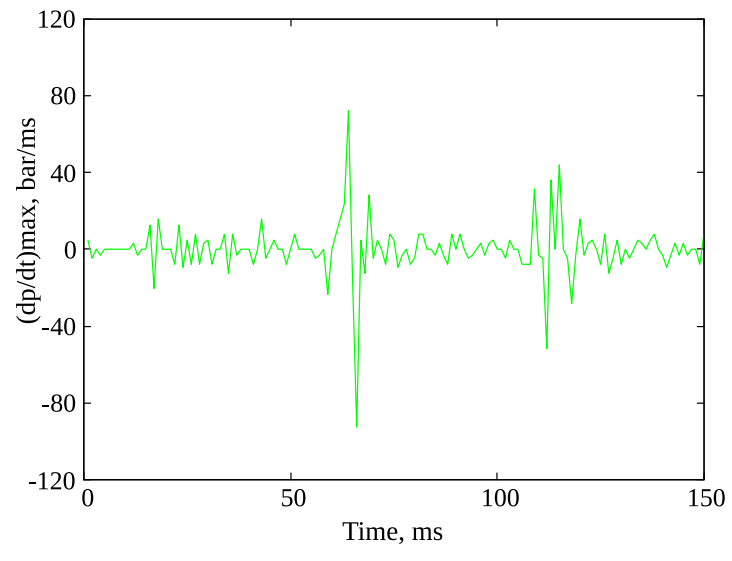

a 2 grams

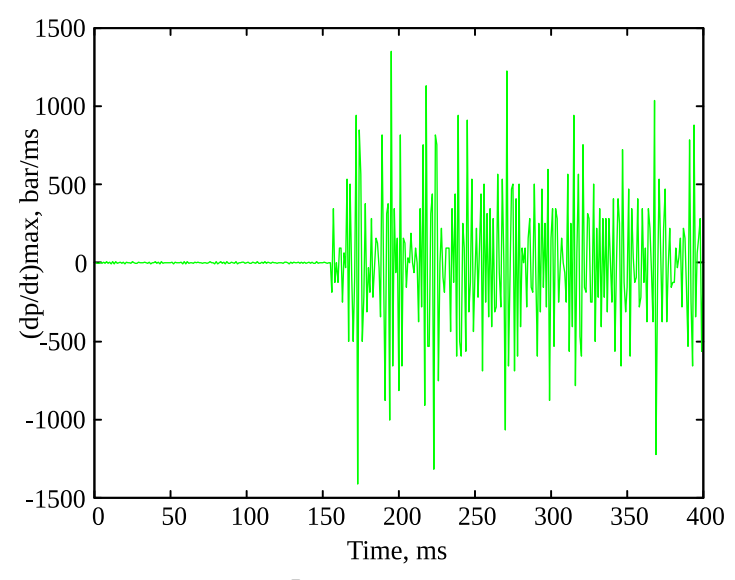

b 5 grams

Fig. 5 Maximum rate of pressure rise $\left(\frac{d p}{d t}\right)_{\max }$ profile for $\mathbf{a} 2 \mathrm{~g}, \mathbf{b} 5 \mathrm{~g}$ 
Fig. 6 Large fireball formation with dust sample of $7 \mathrm{~g}$; A Dust cloud, B Kármán vortex street, C Corrugated flame developed by R-T instability
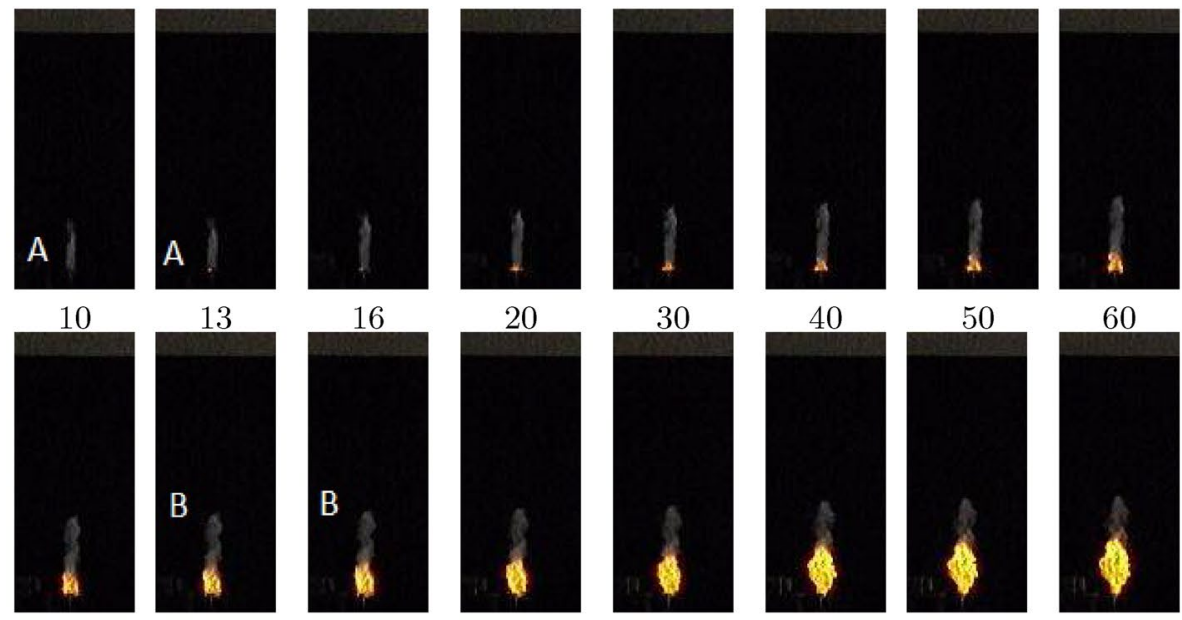

20

30

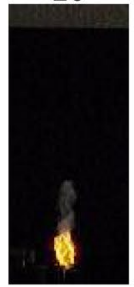

40

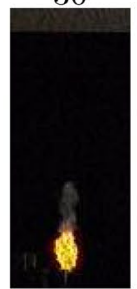

50
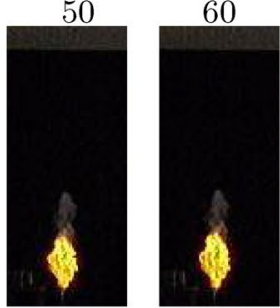

70
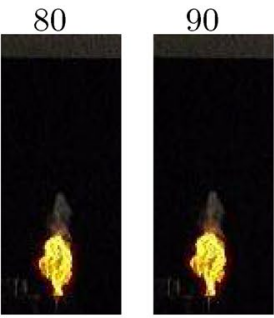

100

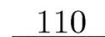

120

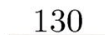

140
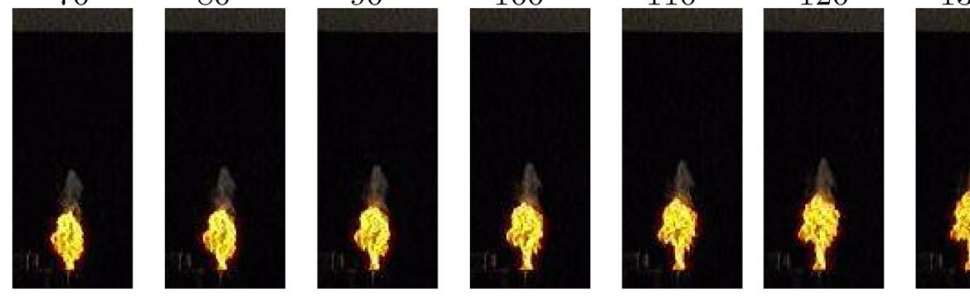

150

160

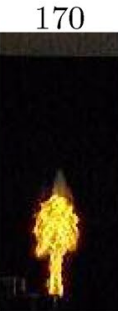

180

190

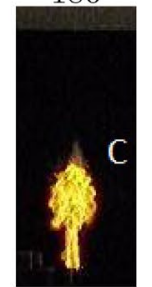

200
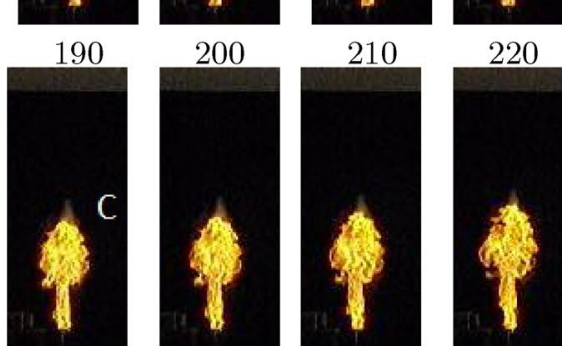

250

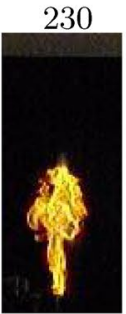

240

260

270

280

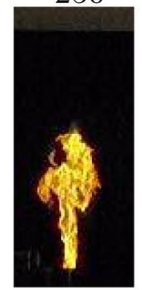

330

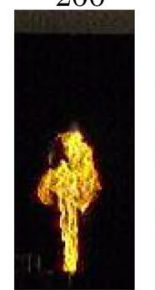

340

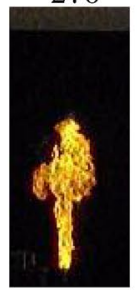

350

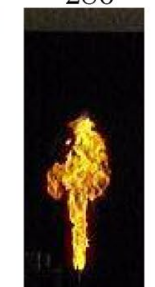

360

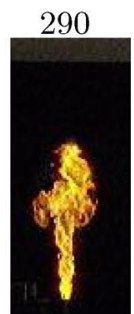

370

380

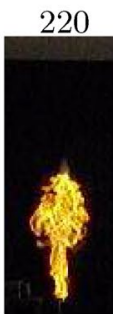

300

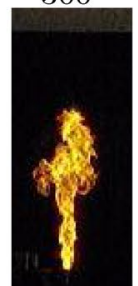

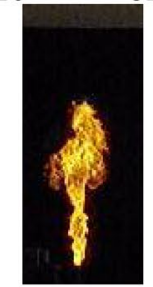

390

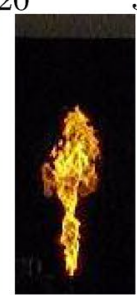

400

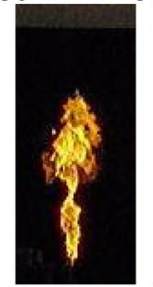

410

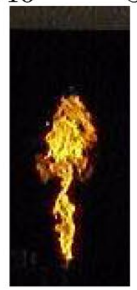

420

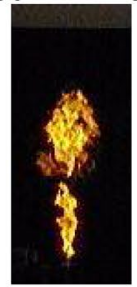

430
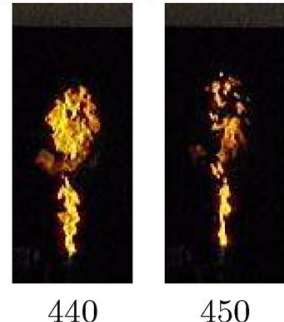
Fig. 7 Stages of flame development in fireball containing $10 \mathrm{~g}$ of dust; A Dust cloud, B Turbulent flame
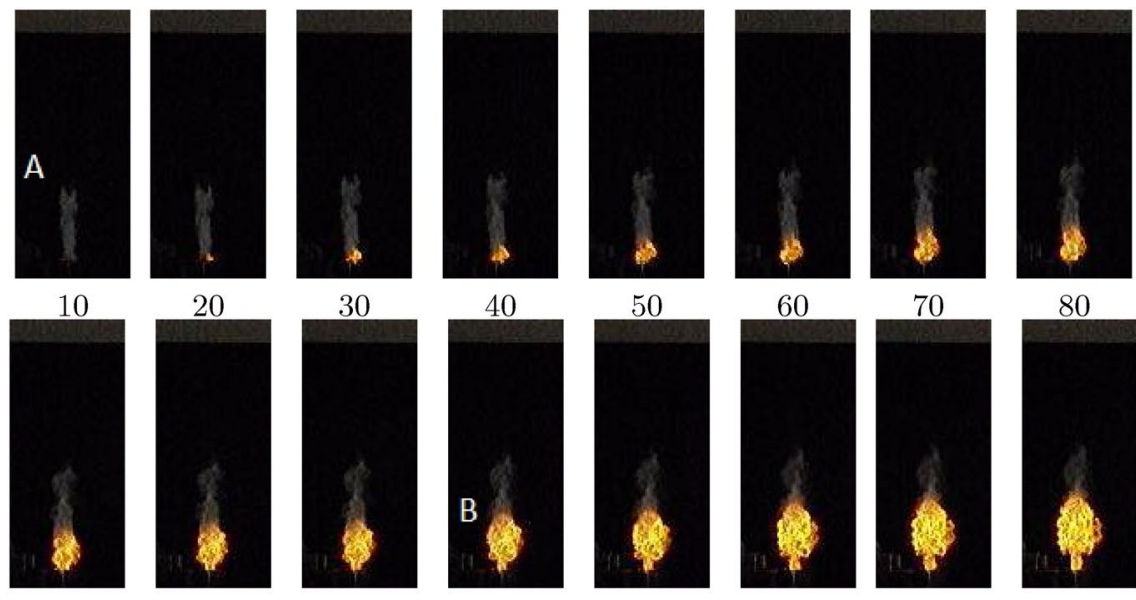

90
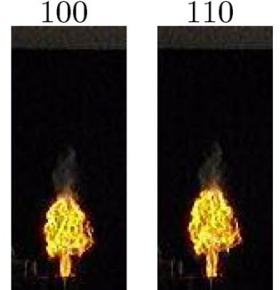

120
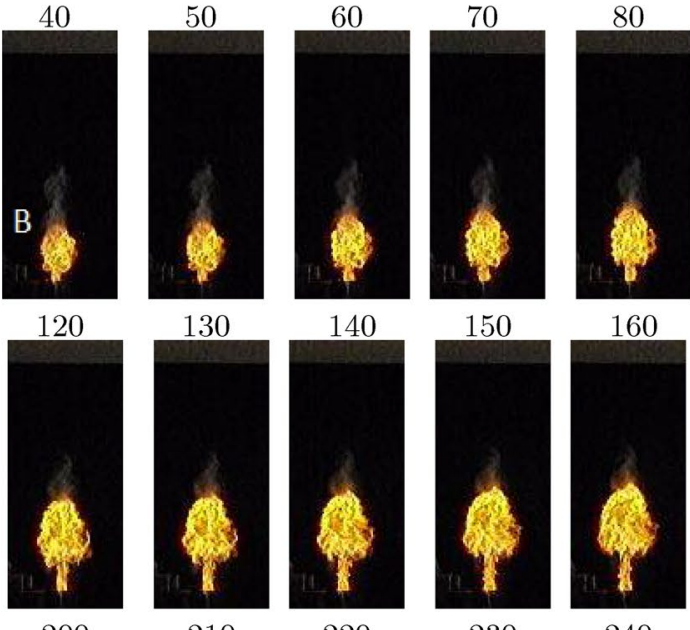

140

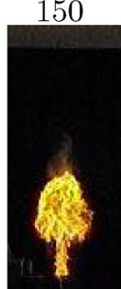

160

210

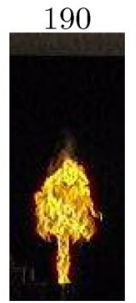

200
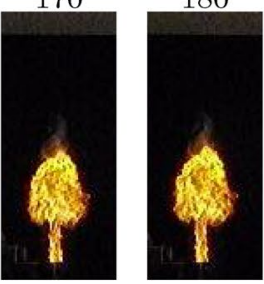

250

260
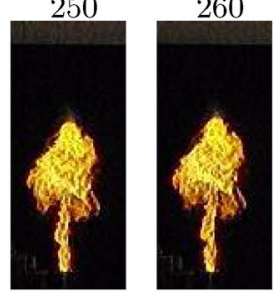

270
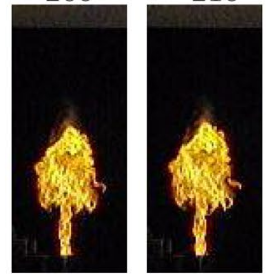

290

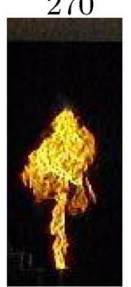

280
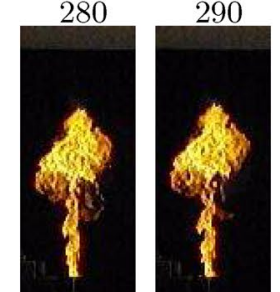

360

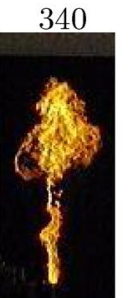

350

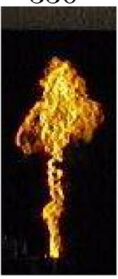

410

420
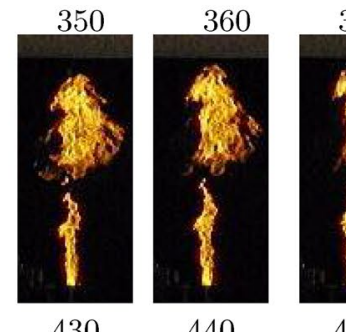

370

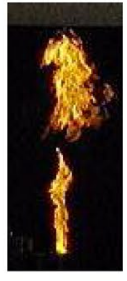

450
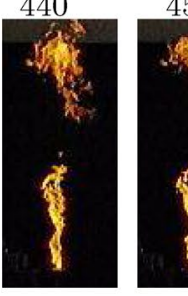

490

500

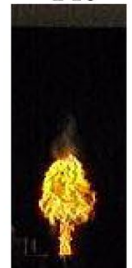

220

230

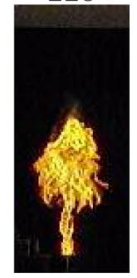

300
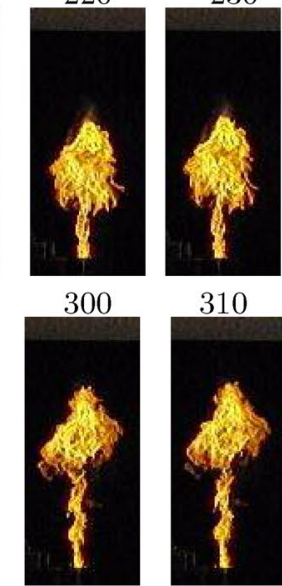

310

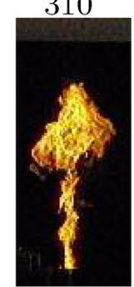

380
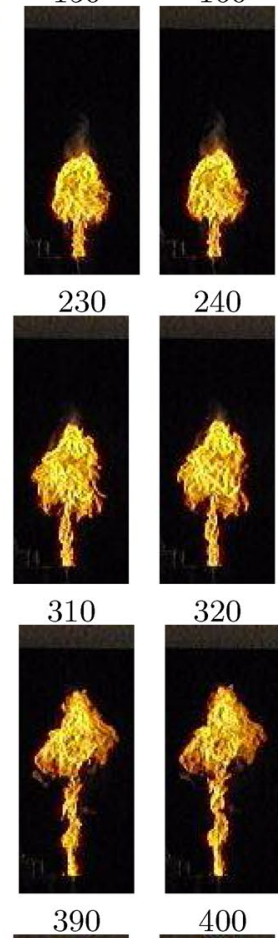

240
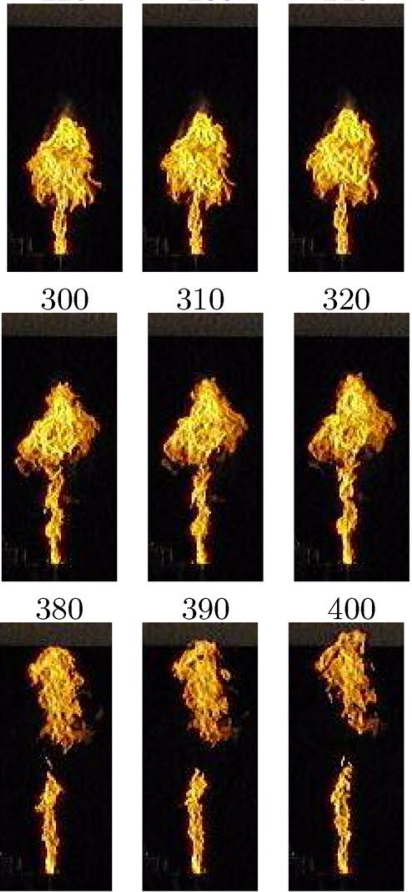

460

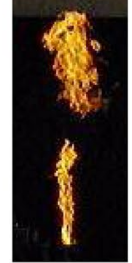

470

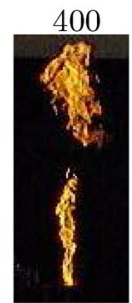

480 
Fig. 8 Corrugated flamelets formed in fireball with dust of $15 \mathrm{~g}$
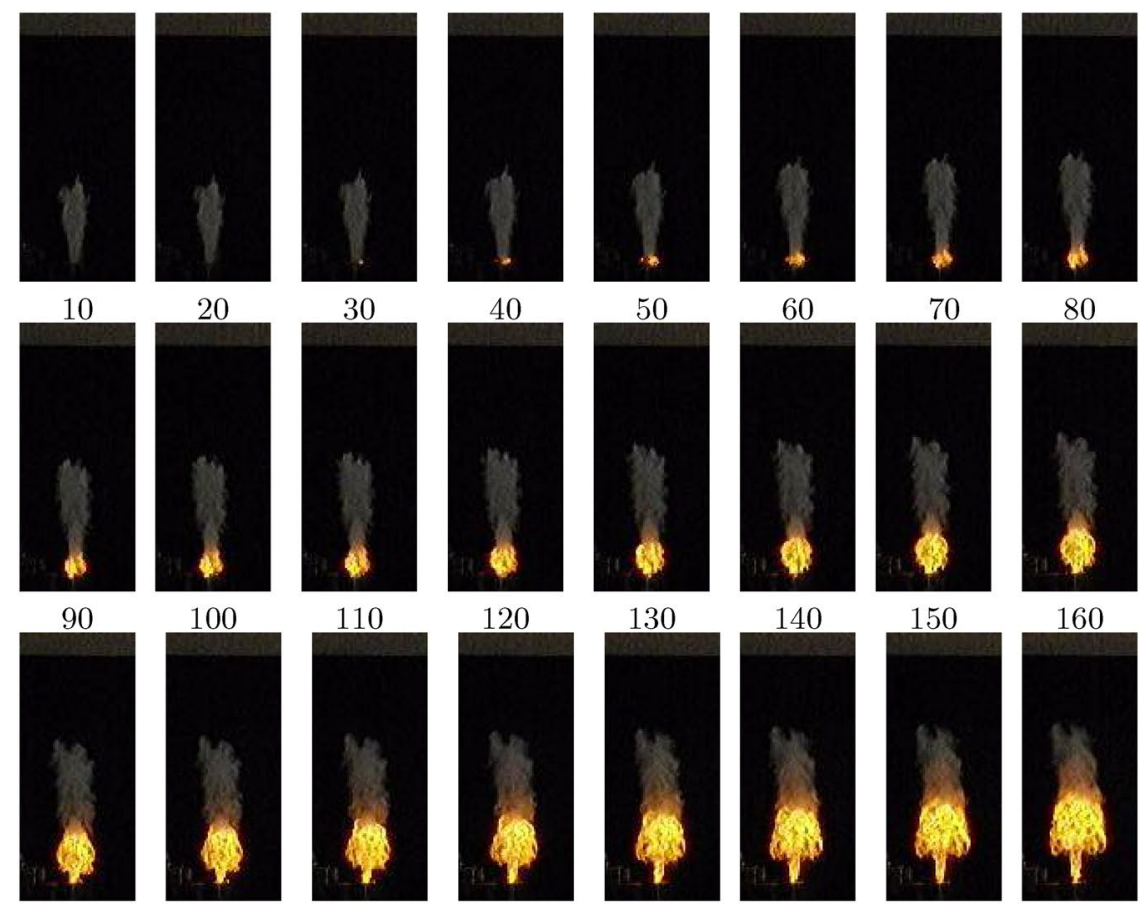

100

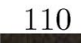

120
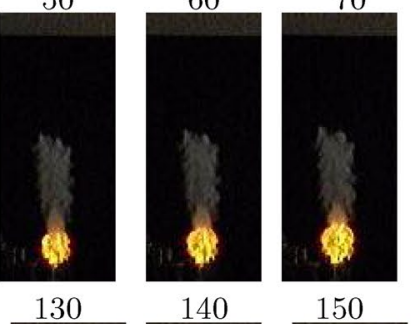

140

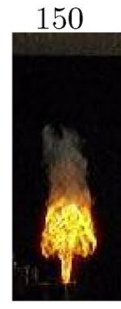

160
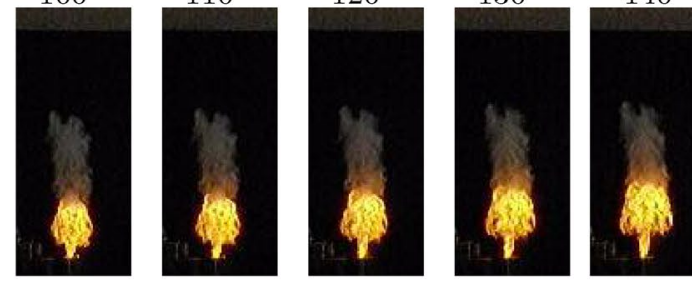

220

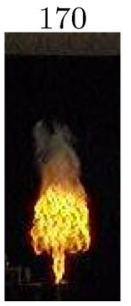

180

190

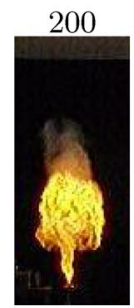

210
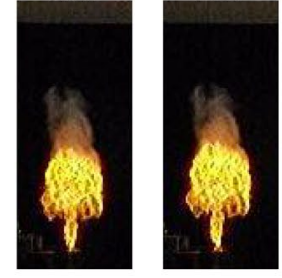

270
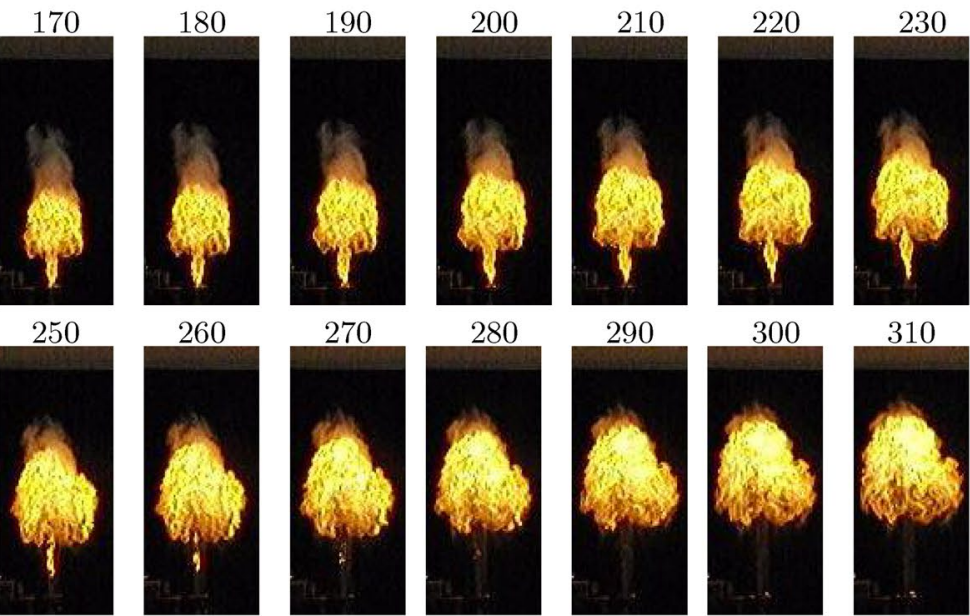

290

300
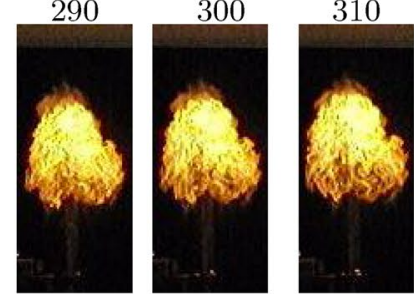

320

330

340

350

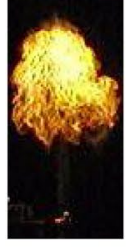

410
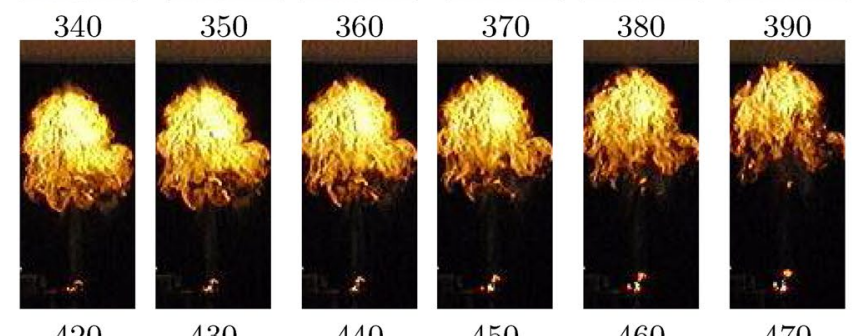

470
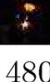
Fig. 9 Surface temperature profile of fireballs

Fig. 10 Wrinkled flame development

Fig. 11 Large turbulent scales in the fireball of different dust samples
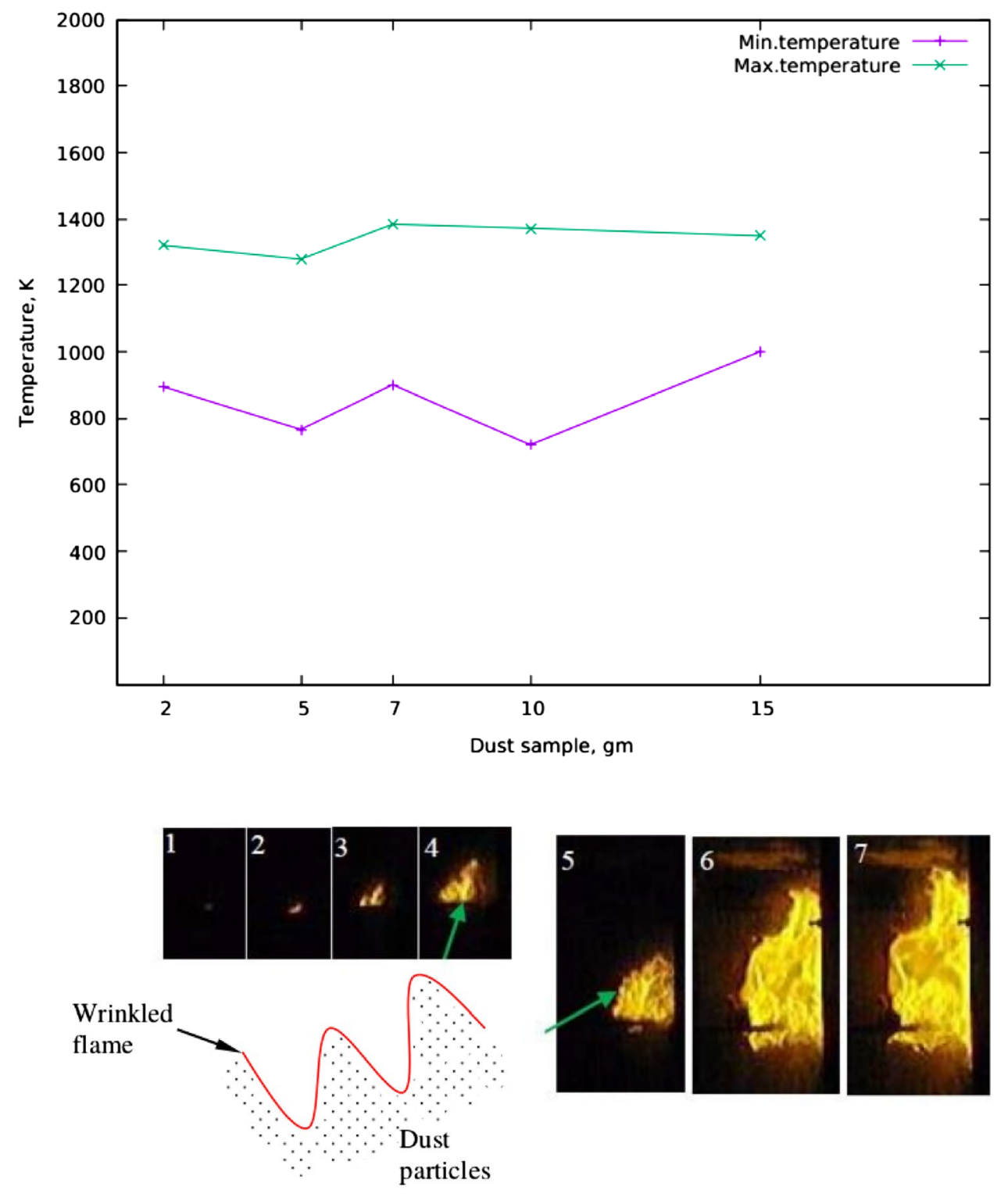

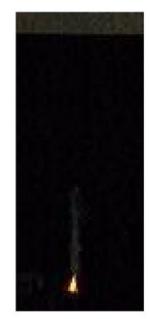

30

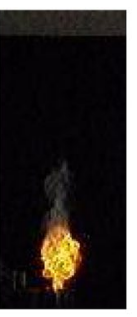

120

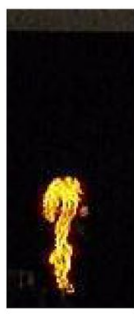

170

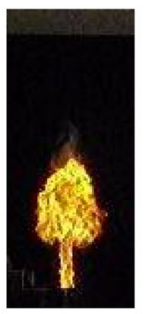

260

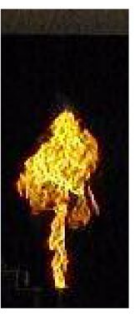

360

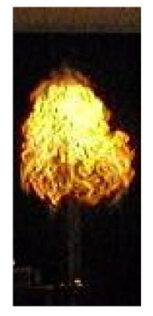

390

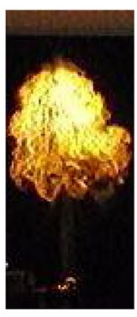

420 

at the interface between flame and surrounding air
Fig. 12 Vortex flow generation

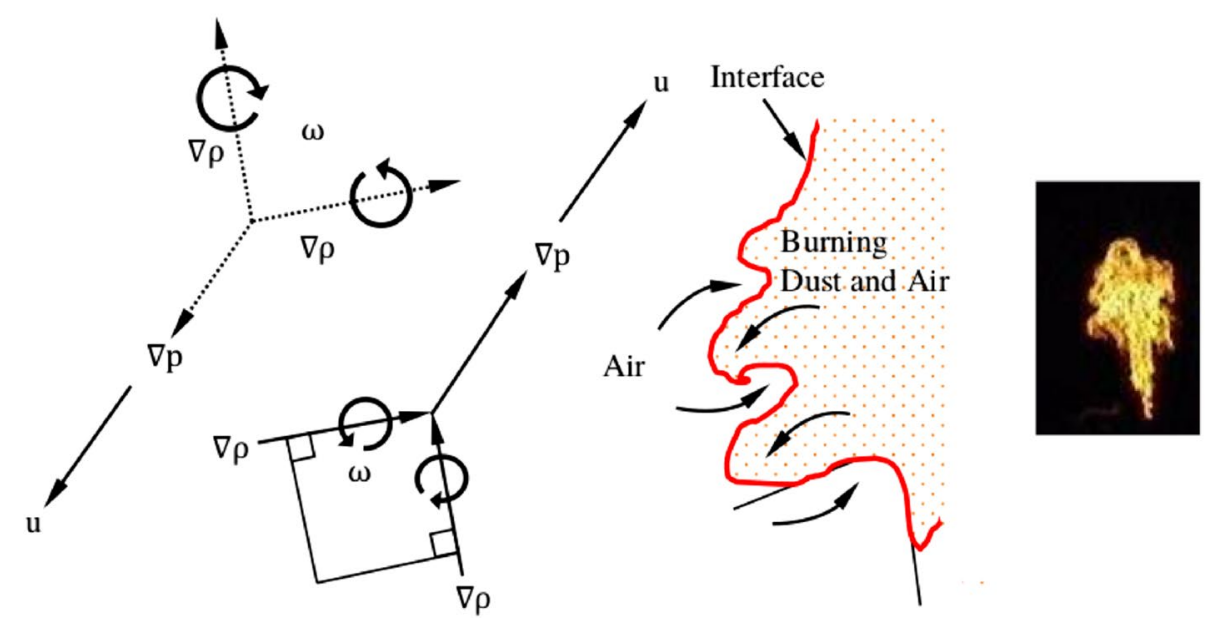

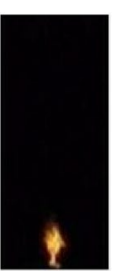

30

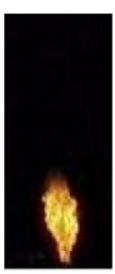

70

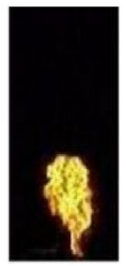

150

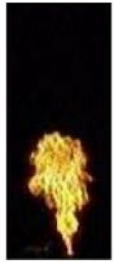

200

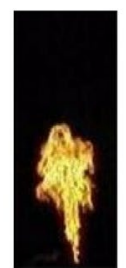

250

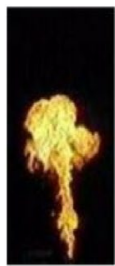

350
Fig. 13 Flame propagation in fireball of corn dust in unconfined (open) space
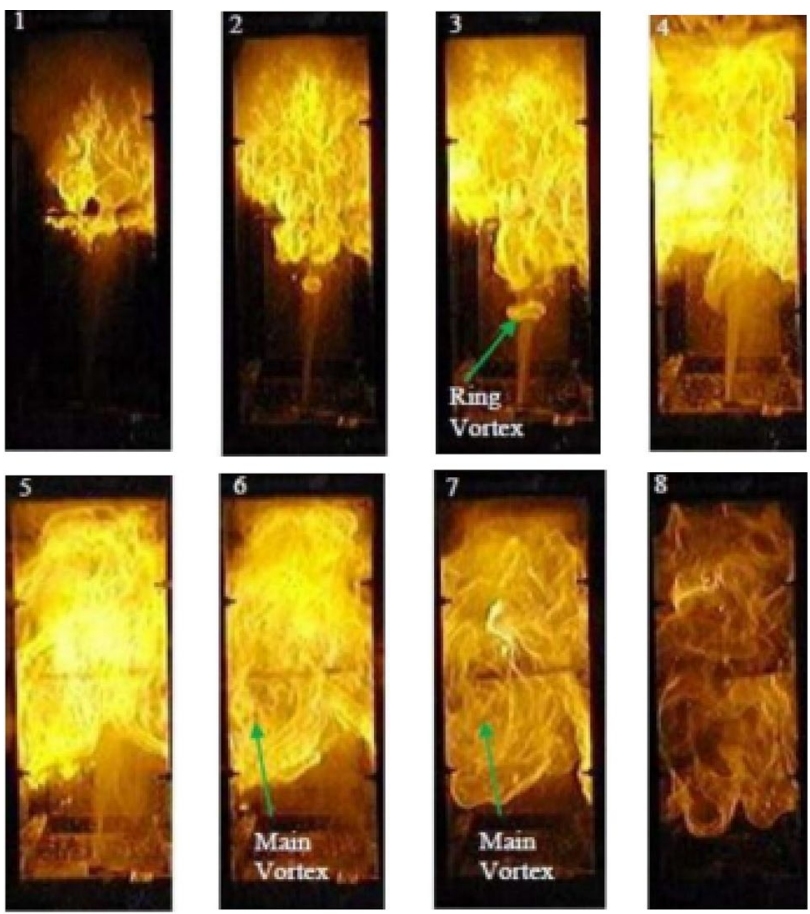

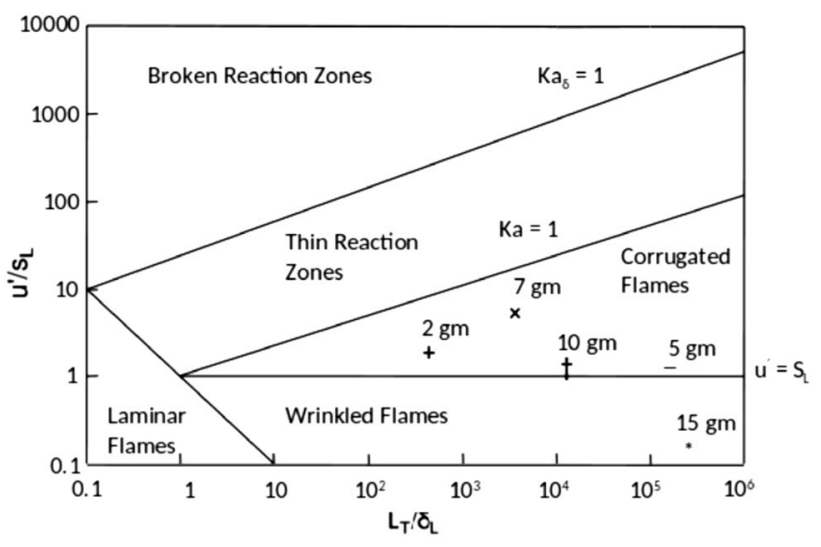

Fig. 15 Borghi-Peters diagram depicting turbulent flame regimes of dust fireballs

lead to explosions. The explosions occurring in about 200 $\mathrm{ms}$ in less than $15 \mathrm{~g}$ of dust can lead to large scale disasters in the flour depots. Thus dust cloud formation should be avoided by passive protection systems like dampers.

\section{Ongoing and future work}

As a continuation to the present work, dust explosions with wheat, rice bran, and husk of the coconut being investigated. The explosion characteristics have been observed to be different for these cases than the cornflour. In addition, 2D and 3D numerical analysis to be conducted to the corn samples to see the complex features at the fireball. So the events which were not noticed in experiments can be brought to the foreground.

Fig. 14 Turbulent Flame in a narrow confined sapce 
Fig. 16 Images arranged in order are $\mathbf{a}$ original color, $\mathbf{b}$ gray and c imcomplemented. Light blue region around dark blue fireball in the imcomplemented image is the preheat zone

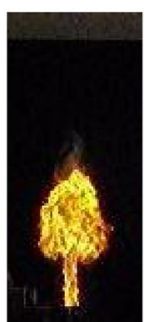

a Color-image

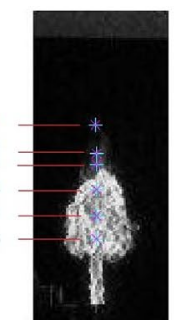

b Gray-image

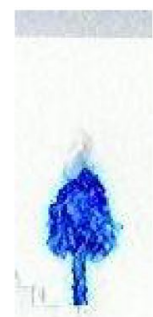

c Imcomplemented-image

\section{Compliance with ethical standards}

Conflict of interest The author declares that there is no conflict of interest.

Similarly, another function "imcrop" was used to confine the image to flame and dust zones.

\section{References}

\section{Appendix}

The image post-processing was done in Matlab software. The aim of image processing is to find the preheat zone temperature. At first, the color image (Fig. 16a) was converted to gray-scale thus the pixel brightness intensity can be evaluated. The intensities range varies between " 0 and 250 ". Then the temperature in the preheat zone could be measured by the reference point where the pixel intensity and the temperature are known. Hence, a pixel intensity of 130 referring $756 \mathrm{~K}$ was taken as the basis from the hot coil data. Now the pixel intensity values were acquired from the Matlab along the axis of the fireball at different points shown in Fig. 16b. At these locations, the temperature was numerically calculated using basis values and the results are given in Table 4. In the Matlab program standard function "impixel ( I, $\mathrm{xi}, \mathrm{yi}$ ) "[35] was used to find pixel intensity. Here, $\mathrm{xi}$ and yi are the coordinates of the pixel in the image (I). Point-1 lies above the fireball in the quiescent air which is at the ambient temperature of $310 \mathrm{~K}$. At point- 2 the temperature is $331 \mathrm{~K}$ which shows that this location is within the preheat zone. Point- 3 is right above the flame surface and its temperature is $389 \mathrm{~K}$. The remaining locations are on the fireball itself, and there the surface temperature reaching as high as $1221 \mathrm{~K}$. The position-5 is in the zone where the dust pocket is at a lower temperature. The color of images was in blue since they are "imcomplemented"[35] in Matlab to find the length of the preheat zone. The light blue region around the fireball is the preheat zone as shown in Fig. 16c.

Matlab function "imrotate" was made use to correct small directional errors which were caused by a misalignment between the camera axis and the horizontal axis.

1. Vorderbrueggen JB (2011) Imperial sugar refinery combustible dust explosion investigation. Process Saf Progress 30(1):66-81

2. Abbasi T, Abbasi SA (2007) Dust explosions cases, causes, consequences, and control. J Hazard Mater 140:7-44

3. Eckhoff RK (1996) Prevention and mitigation of dust explosions in the process industries: a survey of recent research and development. J Loss Prev Process Ind 9:3-20

4. Ogle RA (2017) Dust explosion dynamics. Butterworth-Heinemann, Oxford

5. Yuan Z, Khakzad N, Khan F, Amyotte P (2015) Dust explosions: a threat to the process industries. Process Saf Environ Prot 98:57-71

6. Eckhoff RK (2009) Dust explosion prevention and mitigation, status and developments in basic knowledge and in practical application. Int J Chem Eng. https://doi.org/10.1155/2009/569825

7. Eckhoff RK (2003) Dust explosions in the process industries. Gulf Professional Publishing, Boston

8. Eckhoff RK (2016) Explosion hazards in the process industries. Gulf Professional Publishing, Houston

9. Sweis FK (2020) Dust explosions in the food industries. Int J Sci Eng Invest 9(96):72-77

10. Palazzi E, Curro F, Fabiano B (2020) Low rate releases of hazardous light gases under semi-confined geometry: a consequence based approach and case study application. J Loss Prev Process Ind 63:104038

11. Saeed MA, Andrews GE, Phylaktou HN, Gibbs BM (2017) Flame speed and $K_{\text {st }}$ reactivity data for pulverised corn cobs and peanut shells. J Loss Prev Process Ind 49(Part B):880-887

12. Cashdollar KL (2000) Overview of dust explosibility characteristics. J Loss Prev Process Ind 13:183-199

13. Kuracina R, Szabova Z, Mencik M (2018) Determination of explosion characteristics of sugar dust clouds, transactions of the VSB-Technical University of Ostrava. https://doi.org/10.2478/ tvsbses-2018-0003

14. Zhang Q, Zhang B (2015) Effect of ignition delay on explosion parameters of corn dust/air in confined chamber. J Loss Prev Process Ind 33:23-28

15. Hauge SB, Balakin BV, Kosinski P (2018) Dust lifting behind rarefaction waves. Chem Eng Sci 191:87-99

16. Fedorov AV, Fedorchenko IA (2005) Computation of dust lifting behind a shock wave sliding along the layer. Verification of the model. Combust Explos Shock Waves 41:336-345 
17. Leventiu C, Renou B, Danaila S, Isvoranu D (2016) Accurate measurements and analysis of the thermal structure of turbulent methane/air premixed flame. Energy Proc 85:329-338

18. Sattar H, Andrews GE, Phylaktou HN, Gibbs BM (2014) Turbulent flame speeds and laminar burning velocities of dusts using the ISO $1 \mathrm{~m}^{3}$ dust explosion method. Chem Eng Trans 36:157-162

19. Yoshida A (1981) An experimental study of wrinkled laminar flame. In: Eighteenth symposium (international) on combustion, The Combustion Institute, pp 931-939

20. Thornber B, Drikakis D, Youngs DL, Williams RJR (2010) The influence of initial conditions on turbulent mixing due to Richtmyer-Meshkov instability. J Fluid Mech 654:99-139

21. Thornber B, Drikakis D (2011) Growth of a RichtmyerMeshkov turbulent layer after reshock. Phys Fluids. https://doi. org/10.1063/1.3638616

22. Brouillette M (2002) The Richtmyer-Meshkov instability. Ann Rev Fluid Mech 34(1):445-468

23. Zhou Y (2017) Rayleigh-Taylor and Richtmyer-Meshkov instability induced flow, turbulence and mixing. II. Phys Rep 723-725:1-160

24. Kuhl AIL, Balakrishnan K, Bell JB, Beckner VE (2017) Turbulent combustion in aluminum-air clouds for different scale explosion fields. In: AIP conference proceedings. https://doi. org/10.1063/1.4971730

25. Kim D, Lee SW, Song O-Y, Ko HS (2012) Baroclinic turbulence with varying density and temperature. IEEE Trans Vis Comput Graphics 18:1488-1495

26. Zhang F, Greilich P, Gronig H (1999) Propagation mechanism of dust detonations. Shock Waves 2:81-88

27. Edwards DH, Fearnley PJ, Nettleton MA (1987) Detonation limits of clouds of coal dust in mixtures of oxygen and nitrogen. Combust Explos Shock Waves 23:239-245
28. Cheeda VK, Kumar A, Ramamurthi K (2014) Influence of shear layers on the structure of shocks formed by rectangular and parabolic blockages placed in a subsonic flow-field. Shock Waves 24(2):157-169

29. Cheeda VK, Kumar A, Ramamurthi K (2015) Influence of height of confined space on explosion and fire safety. Fire Saf J 76:31-38

30. Vorobieff $P$, Anderson $M$, Conroy J, White R, Truman CR, Kumar $S$ (2011) Analogues of Rayleigh-Taylor and Richtmyer-Meshkov instabilities in flows with nonuniform particle and droplet seeding. Comput Methods Multiph Flow VI, WIT Trans Eng Sci 70:17-28

31. Dahoe AE, Cant RS, Scarlett B (2001) On the decay of turbulence in the 20 liter explosion sphere. Flow Turbul Combust 67:159-184

32. Bychkov VV, Liberman MA (2000) Dynamics and stability of premixed flames. Phys Rep 325:115-237

33. Li YC, Harbaugh AS, Alexander CG, Kauffman CW, Sichel M (1995) Deflagration to detonation transition fueled by dust layers. Shock Waves 5:249-258

34. Mahmoudi Y, Mazaheri K, Parvar S (2013) Hydrodynamic instabilities and transverse waves in propagation mechanism of gaseous detonations. Acta Astronautica 91:263-282

35. Gonzalez RC, Woods RE, Eddins SL (2017) Digital image processing using MATLAB, 2nd edn. Tata McGraw Hill Education, New York City

Publisher's Note Springer Nature remains neutral with regard to jurisdictional claims in published maps and institutional affiliations. 